\title{
Analysis of Alternatives for Combined and/or Collaborative Syndromic Surveillance Within DoD and VA
}

\author{
Robert E. Bell ${ }^{\star 1}$, Mark Holodniy ${ }^{2}$ and Julie A. Pavlin ${ }^{3}$ \\ ${ }^{1}$ Defense Health Agency, Department of Defense, Falls Church, VA, USA; ${ }^{2}$ Department of Veteran's Affairs, Palo Alto, CA, USA; \\ ${ }^{3}$ Henry M Jackson Foundation and Uniform Services University, Bethesda, MD, USA
}

\section{Objective}

Determine an optimal course of action for achieving a more mission and cost-effective model for implementing combined or collaborative biosurveillance across the Departments of Veterans Affairs (VA) and Defense (DoD).

\section{Introduction}

The Joint Incentive Fund (JIF) Authorization creates innovative DoD/VA sharing initiatives. In 2009, DoD and VA commenced a biosurveillance JIF project whose principle objectives include improved situational awareness of combined VA/ DoD populations 1 and determining the optimal business model allowing both agency biosurveillance programs to operate more efficiently by: 1) consolidating information technology assets; 2) targeting enhanced collaboration for improved public health outcomes; and 3) improving buying power, and return on investment. We analyzed various interoperability models aimed at biosurveillance data sharing, asset consolidation and enhanced collaboration. Potential end states to be evaluated include maintaining separate Departmental systems, bidirectional exchange of data to separately managed systems, consolidation of data within one Department and shared access to a common system, consolidation of data in a neutral repository accessed by separately run legacy systems, or a custom developed biosurveillance solution utilizing a common data repository.

\section{Methods}

Our analysis complied with US Office of Management and Budget's Circular A-94, which promotes efficient resource allocation through well-informed decision-making by providing guidance for conducting cost-benefit and cost-effectiveness analyses. Our analysis utilized the Analytical Hierarchy Process (AHP), which is a decision support methodology for solving complex decision problems using a multi-level hierarchical structure of objectives, criteria, sub-criteria, and alternatives. Pertinent data are derived using a set of pairwise comparisons to obtain weights of importance for decision criteria, and relative performance measures of alternatives for individual decision criterion. Additionally, we used the Delphi technique to solicit input from DoD/VA program leadership, current ESSENCE users, and an independent executive leadership team who formerly served in key positions across both agencies. To assess the merits of current information technology assets, we included a combination of standardized questionnaires as well as "hands on" evaluations and interviews. A wide array of biosurveillance program information was collected from both agencies.

\section{Results}

We identified 6 top level decision criteria and 26 sub-criteria and determined relative importance weighting among this criterion, which formed the basis of an AHP model. Five alternative courses of action as well as the current state were evaluated and scored by public health subject matter experts. These experts scored the alternatives within three discrete timeframes, representing: 1)FY2015-2016; 2)
FY2017-2018; 3)Fy2019-2024. In addition, an overall score was computed for the entire timeframe spanning from FY2015 through 2024. Dual one-way data feeds was the highest scoring alternative for the overall timeframe, however however scores were also very high for the period FY2019-2024, involving the creation of a a Cloud based joint data repository. The DoD and VA will endeavor to pursue these alternatives to improve syndromic biosurveillance efforts in the future.

\section{Conclusions}

This project identified six possible end states for $\mathrm{VA}$ and $\mathrm{DoD}$ future biosurveillance activities. Subject matter experts have determined the criteria which are most important in evaluating alternative scenarios. The two highest scoring solution set's will be pursued in the future in order to improve syndromic biosurveillance across the agencies.

\section{Keywords \\ ESSENCE; Biosurveillance; decision support}

\section{References}

1.Pavlin JA, Burkom HS, Elbert Y, Lucero-Obusan C, Winston CA, et al (2013) Combining Surveillance Systems: Effective Merging of U.S. Veteran and Military Health Data. PLoS ONE 8(12): e84077

\section{${ }^{*}$ Robert E. Bell}

E-mail: robert.bell@dha.mil 